to establish a positive diagnosis. The sequence cough-reflux has also been described but controversy still exist about its prevalence and clinical relevance. One potential factor for such controversy could be due to different methodology for cough detection. The aim of this study was to assess the contribution of cough to pathologic GER in patients with chronic cough and increased esophageal acid exposure.

Methods Simultaneous $24 \mathrm{~h}$ ambulatory reflux and cough monitoring was performed in 17 patients with suspected reflux-related chronic cough. Reflux episodes were detected by $\mathrm{pH}$-impedance monitoring and coughs by a) manometric detection and b) acoustic detection (independent listening of complete $24 \mathrm{hs}$ sound recordings). Manometric cough detection was performed with a twochannel pressure catheter with sensors in the oesophagus and stomach. Cough was manually declared when simultaneous abdomino-thoracic pressure bursts occurred of at least 2 peaks within 3 seconds. Acoustic detection was performed using 3 sound surface sensors. Cough events were identified and inserted into the reflux tracing. We measured total esophageal acid exposure and bolus exposure (by impedance). We calculated the time of acid exposure and bolus exposure after manometrically and acoustic detected cough events.

Results $6 / 17$ patients had pathological esophageal acid exposure. In these patients the total number of reflux events/24hs was 38 (29-70). From that total number, $7.4 \%$ (0-25) reflux episodes occurred within 2 minutes after a cough episodes. Total acid exposure was $9.7 \% \pm 4.7$. From the acid exposure, $3.5 \%$ (0- 8$) \%$ occurred after cough detected by manometry or audio. The median total bolus exposure was $2.1 \pm 0.6 \%$. From total bolus exposure, $6.3 \%$ $(0-20)$ occurred after manometric cough detection and 6.8\% (0-50) after acoustic detected cough. Only $1 / 6$ patients had a significant increase in acid and bolus exposure after cough.

Conclusion In patients with chronic cough and pathological acid GER, cough was followed by reflux in a minority of cases regardless of the method for cough detection. In only $1 / 6$ patients the sequence cough-reflux could be considered as causal of pathological GER.

Disclosure of Interest None Declared

\section{PTU-131 OESOPHAGEAL THERMAL SENSITIVITY AND MUCOSAL INTEGRITY IN HEALTHY SUBJECTS. A STUDY USING A NOVEL PELTIER-BASED HEATING DEVICE AND IMPEDANCE}

doi:10.1136/gutjnl-2013-304907.221

1." M Al-Zinaty, 'P Woodland, ${ }^{2} \mathrm{~J}$ Reeves, ${ }^{2} \mathrm{M}$ Birch, ${ }^{1} \mathrm{Q}$ Aziz, 'D Sifrim. ${ }^{1}$ Barts \& The London School of Medicine, Queen Mary University of London; ${ }^{2}$ Clinical Physics CAU, Barts Health NHS Trust, London, UK

Introduction Investigation of oesophageal sensitivity might help better improve phenotyping of patients with GERD, functional heartburn and non-obstructive dysphagia. Studies on oesophageal thermal sensitivity have used balloon techniques with circulating water. This lacks linear control of heating, has relative heat loss, and provokes mechanical stimulation by the distended balloon. Oesophageal mucosal integrity is important in symptoms perception and can be studied using impedance "in vivo". We developed a catheter device incorporating a "thermal stimulator" using Peltier heating technology and impedance electrodes to assess mucosal contact and integrity. We aimed to investigate oesophageal heat sensation/pain thresholds and their relationship with mucosal integrity in healthy human subjects.

Methods 21 healthy volunteers underwent thermal stimulation using an oesophageal catheter with a $7 \mathrm{~mm}$ electronic Peltier heater, to deliver a ramp heating protocol up to $60^{\circ} \mathrm{C}$ at 5 and $15 \mathrm{~cm}$ above LOS. Symptoms were recorded by computerised Visual Analogue Score (VAS). The delivered temperature at Pain Detection Threshold (PDT), time to PDT was measured and the area under the heating curve (AUC) was calculated. Oesophageal impedance was assessed over 10 minutes pre (basal), during and post heating in the distal and proximal oesophagus. The protocol was repeated after 2 weeks to assess reproducibility.

Results Temperature at PDT showed a very low SD in the distal and proximal oesophagus and Bland-Altman Tests showed good reproducibility $(-0.190595 \% \mathrm{CI}-1.4132$ to 1.0327$)$. There were no significant differences in thermal sensitivity parameters between distal and proximal oesophagus. Due to the low inter-individual

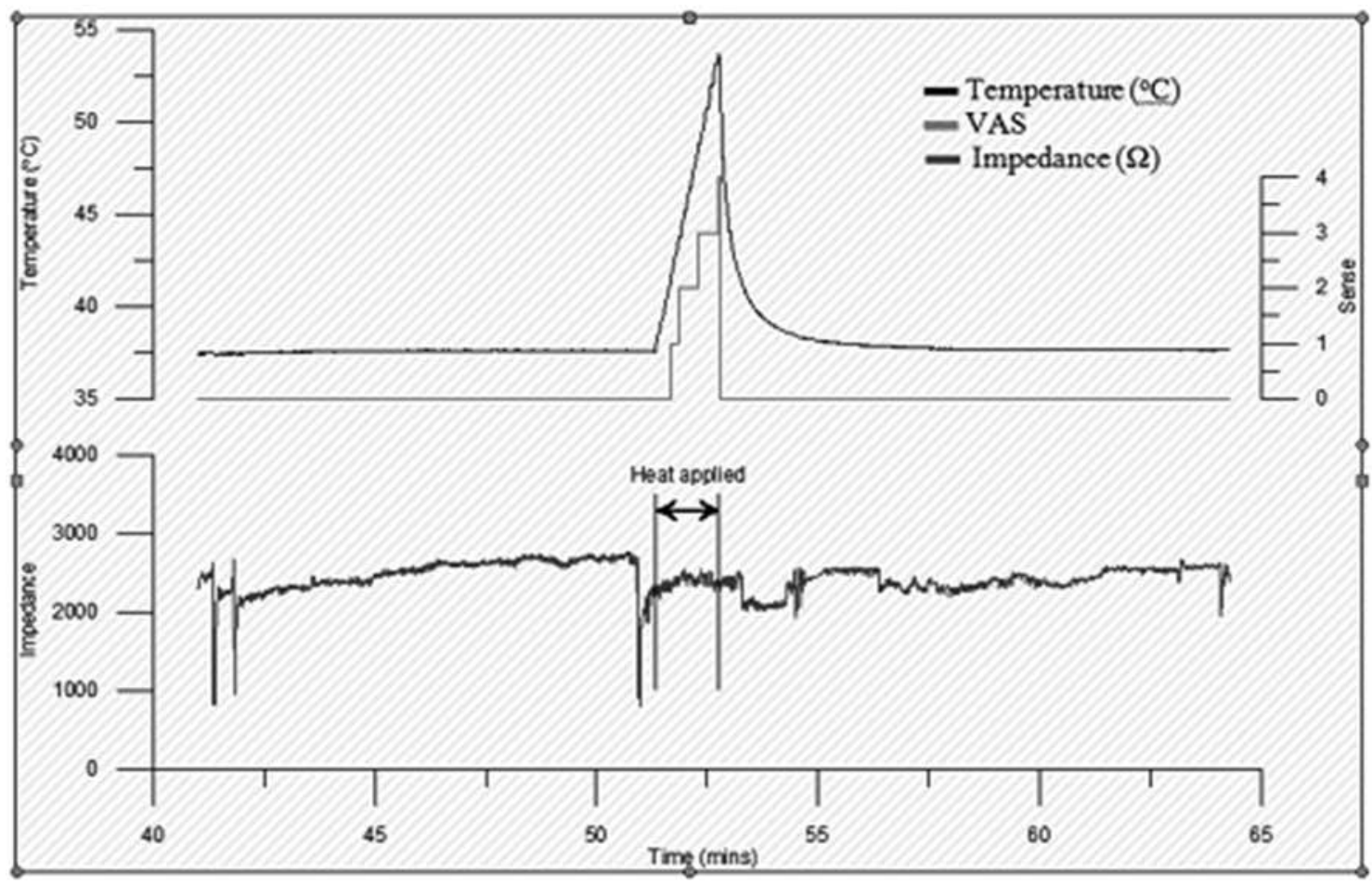

Abstract PTU-131 Figure 
variability in thermal sensitivity between healthy subjects, we could not find a significant correlation between impedance and perception parameters in the proximal or distal oesophagus.

\section{Abstract PTU-131 Table 1}

\begin{tabular}{llll}
\hline & Temperature at PDT $\left({ }^{\circ} \mathbf{C}\right)$ & AUC $\left({ }^{\circ} \mathbf{C} * \mathbf{s}\right)$ & Time to PDT $(\mathbf{s})$ \\
\hline Distal Oesophagus & $53.7 \pm 2.7$ & $698.8 \pm 241.3$ & $85.1 \pm 15.5$ \\
Proximal Oesophagus & $54.3 \pm 2.3$ & $743.5 \pm 201.9$ & $87.8 \pm 11.7$ \\
\hline
\end{tabular}

Conclusion A novel Peltier-based thermal stimulator device can accurately and reproducibly determine oesophageal thermal sensitivity. In healthy subjects we could not demonstrate a correlation between basal impedance and sensitivity to heating. Current experiments are assessing this correlation in NERD patients with hypersensitivity to acid.

Disclosure of Interest None Declared

\section{PTU-132 CARBONATED SOLUTIONS ARE SUPERIOR TO SOUR SOLUTIONS IN MODIFYING HUMAN SWALLOWING REACTION TIME PERFORMANCE}

doi:10.1136/gutjnl-2013-304907.222

1."0 Elshukri, 'E Michou, 'S Mistry, 'S Hamdy. 'Gastrointestinal Sciences, Institute of Inflammation and Repair, University of Manchester, Manchester, UK

Introduction Background/Aims Clinical evidence supports a role for carbonated liquids in reducing aspiration in dysphagic patients compared to simple water (Bulow et al Acta Radiologica, 2003). However, there are limited data on how carbonation modulates swallowing performance. Here, we investigate the effects of equi-pH carbonated and sour (citric acid) water solutions on swallowing performance using a reaction time task (Mistry et al J Physiol.2007), in healthy volunteers.

Methods Twelve healthy participants ( 6 male, $33 \pm 4$ years, mean \pm SEM) visited the laboratory on three separate occasions. Subjects were asked to perform 5 consecutive measurements of swallowing behaviour with 10 normal,10 fast and 10 challenged swallows per measurement (within a pre-determined time-window). For each task, subjects swallowed each of three solutions: carbonated, sour/ citric acid and still water solutions with each attendance in a randomised order. The 5 blocks of 30 swallows were repeated over a 1 hour period at 0 15, 30, 45 and 60 minutes to control for fatigue and practise confounding variables. Measurements were performed through an intra-pharyngeal catheter with built-in pressure transducers to record change in pharyngeal pressure. A pair of electrodes on the back of subject's hand was used to deliver an electrical pulse to cue the subject when to swallow. Subjects were invited for an additional visit to complete a 'taste intensity' questionnaire. Data were analysed with non-parametric Wilcoxon's test in SPSS16.

Results Mean swallowing latencies of both normal and fast swallows were not significantly different across the three different sessions. However, for the challenged swallowing task, compared to still water, carbonated water significantly improved the number of correct swallows $(Z=-2.044, p=0.041)$. By contrast, sour (citric acid) solutions had no effects on challenged swallowing compared water [citric acid*water $(Z=-0.045, P=0.9640)]$, despite being similar to carbonated water [citric acid*carbonated $(Z=-1.293$, $p=0.196)]$. Carbonated solutions also had the highest intensity score being greater than both sour and still water [carbonated*water $(Z=-3.517, P=0.000)$, carbonated *citric acid $(Z=-3.520$, $\mathrm{p}=0.000)]$

Conclusion Carbonation appears to alter swallowing performance compared to other liquids by improvement in complex tasks and showed greatest perceived taste intensity. These data support the using of carbonation as treatment option for dysphagic patients in preventing aspiration and lay the basis for further clinical research with carbonation on swallowing function.

Disclosure of Interest None Declared

\section{REFERENCES}

1. Bulow et al. Acta Radiologica., 2003

2. Mistry et al. J Physiol., 2007

\section{PTU-133 GOBLET CELL CARCINOMA OF THE APPENDIX: ACUTE VS CHRONIC PRESENTATIONS}

doi:10.1136/gutjnl-2013-304907.223

1.* R Basuroy, ${ }^{1} \mathrm{~A}$ Ngu, ${ }^{2} \mathrm{~L}$ Mills, ${ }^{3} \mathrm{H}$ El-Mahallawi, ${ }^{4} \mathrm{~T}$ Cecil, ${ }^{1,2} \mathrm{~J} \mathrm{~K}$ Ramage. ${ }^{1}$ Department of Gastroenterology, Hampshire Hospitals NHS Trust, Basingstoke; ${ }^{2}$ Neuroendocrine Tumour Service, Institute of Liver Studies, Kings College Hospital, London; ${ }^{3}$ Department of Pathology; ${ }^{4}$ Pseudomyxoma peritonei Service, Hampshire Hospitals NHS Trust, Basingstoke, UK

Introduction Goblet Cell Carcinomas (GCC) are a rare neuroendocrine tumour (NET) of the appendix. The experience of a National Specialist Centre's for pseudomyxoma peritonei (cytoreductive surgery) and two regional NET services with patients with GCC are presented.

Methods A retrospective audit was performed of patients with histologically proven GCC collated from histology, NET and cytoreductive surgery databases. Mortality rates (\%) for subgroups were calculated.

Results 16 patients (female $=9$ ) were included for analysis with median age at diagnosis of 58 years (range, 25.5-71.8). The mortality rate was $25 \%(n=4)$ associated with median survival of 22 months (range, 9-72) following diagnosis. The most common symptoms were acute appendicitis-like (62.5\%), chronic abdominal pain (50\%), bowel obstruction (25\%) and chronic diarrhoea (12.5\%). Patients presenting with acute appendicitis-like symptoms had the lowest mortality rate at $10 \%$. Bowel obstruction, chronic abdominal pain and chronic diarrhoea were associated with mortality rates of $50 \%, 38 \%$ and $50 \%$ respectively. Completion right hemicolectomy $(\mathrm{n}=10)$ was associated with decreased mortality $(22 \%$ vs $40 \%)$. Bilateral salphingo-oophrectomy $(n=5)$ was associated with increased mortality ( $66 \%$ vs $0 \%$ ). The mortality rate associated with chemotherapy $(n=6)$ and cytoreductive surgery $(n=4)$ was $17 \%$ and $50 \%$ respectively.

Conclusion Patients with GCC who present acutely have better outcomes than those with chronic symptoms suggesting different disease processes. Performing a completion right hemicolectomy is associated with benefit, while the role of BSO is less clear.

Disclosure of Interest None Declared

\section{PTU-134 PREVALANCE AND INVESTIGATIONAL PATHWAYS OF PATIENTS WITH CONSTIPATION PREDOMINANT IRRITABLE BOWEL SYNDROME}

doi:10.1136/gutjnl-2013-304907.224

1.'S Lin, 'M Kurien, 'I Aziz, 'S Winfield, 'N Rugg, 'A Kelsall, '1J S Leeds, 'D S Sanders. 'Department of Gastroenterology, Royal Hallamshire Hospital, Sheffield, UK

Introduction Our group has previously described investigational pathways that occur in patients with diarrhoea predominant irritable bowel syndrome (IBS-D). Currently, there is a paucity of work undertaken in either primary or secondary evaluating patients with constipation predominant irritable bowel syndrome (IBS-C). This study evaluates the population prevalence of IBS-C, determining also the investigational pathways that occur in these patients.

Methods We prospectively collected data from 3 groups of patients between April 2005 and November 2012. Group 1 ( $n=1002)$ were healthy volunteers, Group $2(n=64)$ were patients fulfilling Rome III criteria for IBS-C, with Group $3(n=403)$ being patients fulfilling 